\title{
The Contribution of Psychological Distress to Resting Palpitations in Patients Who Recovered from Severe COVID-19
}

\author{
Bing Huang ${ }^{1-4}$ \\ Hui Yan ${ }^{2}$ \\ Limei $\mathrm{Hu}^{1,3,4}$ \\ Guiqiu Cao $\mathbb{D}^{2}$ \\ Guipeng Wang ${ }^{2}$ \\ Jing Meng ${ }^{2}$ \\ Wanting $\mathrm{Li}^{5}$ \\ Gang Liu ${ }^{2}$ \\ Juan Wang ${ }^{2}$ \\ Wenqing Le ${ }^{5}$ \\ Hong Jiang ${ }^{1,3,4}$ \\ 'Department of Cardiology, Renmin \\ Hospital of Wuhan University, Wuhan, \\ Hubei, People's Republic of China; \\ ${ }^{2}$ Department of Cardiology, Fifth \\ Affiliated Hospital of Xinjiang Medical \\ University, Urumqi, Xinjiang, People's \\ Republic of China; ${ }^{3}$ Cardiovascular \\ Research Institute, Wuhan University, \\ Wuhan, Hubei, People's Republic of \\ China; ${ }^{4}$ Hubei Key Laboratory of \\ Cardiology, Wuhan, Hubei, People's \\ Republic of China; ${ }^{5}$ Department of \\ Critical Care, Wuhan Hankou Hospital, \\ Wuhan, Hubei, People's Republic of \\ China
}

Correspondence: Hong Jiang

Department of Cardiology, Renmin Hospital of Wuhan University, No. 238 jiefang Road,

Wuchang District, Wuhan City, Hubei

Province, 430060, People's Republic of China

Tel +86 278804191 I

Fax +862788042292

Email hong-jiang@whu.edu.cn

Guiqiu Cao

Department of Cardiology, Fifth Affiliated Hospital of Xinjiang Medical

University, No. 118 Henan Road, Xinshi

District, Urumqi, Xinjiang, 830000 ,

People's Republic of China

$\mathrm{Tel} / \mathrm{Fax}+8609917598403$

Email 809624056@qq.com
Background: Increasing numbers of patients have recovered from severe coronavirus disease 2019 (COVID-19) in Wuhan, China. This study aimed to evaluate the association of psychological distress with resting palpitations in recovered patients.

Methods: In this prospective cohort study, consecutive patients who recovered from severe COVID-19 and complained of resting palpitations were included. Dynamic electrocardiogram (ECG) was continuously monitored for 2 hours while patients were at rest. A survey using a palpitation frequency scale and the Hospital Anxiety and Depression Scale (HADS) was administered to all participants.

Results: Of the 289 consecutive patients who recovered from severe COVID-19, 24 patients $(8.3 \%)$ suffered resting palpitation symptoms, and 22 patients were finally included. Twohour Holter monitoring showed that $18(81.8 \%)$ patients had tachyarrhythmias, of which the most common was sinus tachycardia $(17 / 22,77.3 \%)$. However, patients with sinus tachycardia showed a similar frequency of palpitation episodes compared to those without sinus tachycardia. Anxiety (68.2\%) and depression (59.1\%) were prevalent among these recovered patients. Patients with anxiety or depression symptoms had a higher frequency of palpitation episodes than those without anxiety or depression symptoms. In addition, both the HADSanxiety score $(r=0.609, P<0.01)$ and HADS-depression score $(r=0.516, P=0.01)$ were positively related to the frequency of palpitation episodes.

Conclusion: Symptoms of resting palpitations, manifested mainly by sinus tachycardia, are not uncommon in patients who recovered from severe COVID-19. Psychological distress (anxiety and depression) may be responsible, at least in part, for resting palpitation symptoms.

Keywords: coronavirus disease 2019, palpitations, sinus tachycardia, anxiety, depression

\section{Introduction}

An increasing number of patients recover from COVID-19 and are discharged from hospitals. However, approximately half of recovered COVID-19 patients still suffer from various clinical symptoms, ${ }^{1}$ and symptoms of resting palpitations are not rare. Cardiovascular complication, especially myocardial injury, is uncommon among patients with severe COVID-19. ${ }^{2}$ However, current studies show that myocardial injury is not correlated with the symptoms of chest tightness and palpitations, not only during hospitalization but also after discharge. ${ }^{3}$ So far, the causes of palpitation symptoms complicated COVID-19 remain unclear.

COVID-19 pandemic has imposed significant level of psychological burden worldwide. ${ }^{4}$ Several studies have focused on mental health in COVID-19 patients. 
The results found that a majority of the patients with COVID-19 experienced various psychological symptoms during hospitalization and even after discharge. ${ }^{5}$ Anxiety and depression are common mental health problems in COVID-19 patients, especially in severe cases associated with worse outcomes and longer hospital stays. ${ }^{6}$ It is well accepted that psychological distress is one of the most common causes of palpitation symptoms and is associated with symptom severity. ${ }^{7,8}$ Therefore, we speculate that psychological distress might also contribute to the resting palpitation symptoms in patients recovering from COVID-19.

Measures of psychological distress can complement medical assessment in the diagnosis of patients with palpitations. ${ }^{9}$ However, few studies have paid attention to psychological distress in recovered COVID-19 patients with resting palpitations. The aim of the present study was to investigate the prevalence of anxiety and depression and their association with the frequency of palpitation episodes in patients who recovered from severe COVID19 and complained of resting palpitations.

\section{Methods}

\section{Participants}

This single-centre, prospective, cohort study was performed at Hankou Hospital in Wuhan City, China. Consecutive patients who recovered from severe COVID-19 and complained of resting palpitation symptoms from February 1 to April 1, 2020, were included.

COVID-19 was confirmed and diagnosed by Nucleic acid testing of SARS-CoV-2 RNA in nasal/oropharyngeal swab samples. Severe cases of COVID-19 were defined by the presence of any of the following: ${ }^{10}$ shortness of breath and respiratory rate $>30$ breaths/min; oxygen saturation $\leq 93 \%$; partial pressure of oxygen to fraction of inspired oxygen ratio $\leq 300 \mathrm{mmHg}$; lung infiltrates $>50 \%$ within 24 to 48 hours; respiratory failure; septic shock; and/or multiple organ dysfunction or failure. All recovered COVID-19 patients met the discharge criteria as follows: ${ }^{10}$ afebrile for at least three days, significantly improved respiratory symptoms, improvement in radiological abnormalities on chest radiograph or $\mathrm{CT}$, and two consecutive negative SARSCoV-2 tests that were more than 24 hours apart. Patients who refused to participate in this study were excluded.

The study protocol was approved by the ethics committees of Wuhan Hankou Hospital (WHHES-2020-0201).
Written informed consent was obtained from all the individuals who participated in this study.

\section{Data Collection}

Patient data, including demographics, medical history, clinical manifestations, laboratory examinations, comorbidities, complications, and hospital stay, were collected from the electronic medical records by a trained team of physicians. All the data were independently reviewed. Acute respiratory distress syndrome was defined according to the Berlin definition. ${ }^{11}$ Acute myocardial injury was defined as an increase in the blood level of the cardiac biomarker cTnI above the 99th percentile upper reference limit. ${ }^{12}$ Acute liver injury was defined as liver enzymes and/or bilirubin more than twice the upper reference limit. ${ }^{12}$ Acute kidney injury was identified according to the Kidney Disease: Improving Global Outcomes definition. ${ }^{13}$ Coagulopathy was defined as a 3-s extension of prothrombin time or a 5-s extension of activated partial thromboplastin time. ${ }^{12}$

\section{Electrocardiogram (ECG) Monitoring}

Twelve-lead ECG monitoring was continuously performed for 2 hours using a Holter monitor (CT-86, Baihui, Hangzhou City, China) in all enrolled participants before they were discharged. All patients lied on the bed quietly with no drink or food for 15 minutes before the monitoring until completion of the monitoring. Holter software was used to analyse the heart rate and arrhythmias. Sinus tachycardia was defined as an increase in sinus rhythm to $>100$ beats/ min. Atrial tachycardia was defined as 3 or more consecutive atrial premature contractions. All ECG data were manually examined by two blinded observers and arbitrated by electrophysiologists for any suspicious event classification.

\section{The Frequency of Palpitation Episodes Scale}

All participants completed a 7-point rating scale of the frequency of their palpitation episodes as follows: $1=$ rarely (approximately once or twice a day), 2=sometimes (3-5 times a day), $3=$ more than 5 times a day but less than once an hour, 4=approximately once or twice an hour, 5=3-5 times an hour, $6=$ more than 5 times an hour but not sustained, and $7=$ sustained. A higher palpitation score indicates a higher frequency of palpitation episodes. 


\section{The Hospital Anxiety and Depression Scale (HADS)}

The HADS is a widely used scale for determining anxiety (HADS-A) and depression (HADS-D) in the clinical setting. ${ }^{14}$ It comprises 14 items with a score of 0 to 3 for each item. There was a total score of 21 for anxiety (7 items) and depression (7 items). The cut-off for clinical anxiety or depression was defined as HADS-A or HADSD score $\geq 8$, respectively. A higher score indicates greater anxiety or depression levels.

\section{Statistical Analysis}

The continuous and categorical variables are represented as the median (interquartile range $[\mathrm{IQR}]$ ) and as counts (percentage), respectively. The means for continuous variables were compared using independent-sample $t$-tests when the data were normally distributed; otherwise, the Mann-Whitney test was used. The associations of maximum heart rate, HADS-A score, and HADS-D score with palpitation score were analysed by Pearson's correlation. All data were analysed using SPSS Statistics for Windows, version 20 (IBM Corp., Armonk, NY, USA). The differences were considered statistically significant at a $P$ value of $<0.05$ (two-sided).

\section{Results}

\section{Patient Characteristics}

A total of 289 consecutive patients who recovered from severe COVID-19 were screened initially from February 1 to April 1, 2020. Figure 1 shows a flowchart for patient recruitment. Of these patients, 24 patients $(8.3 \%)$ suffered different degrees of palpitation symptoms. After excluding

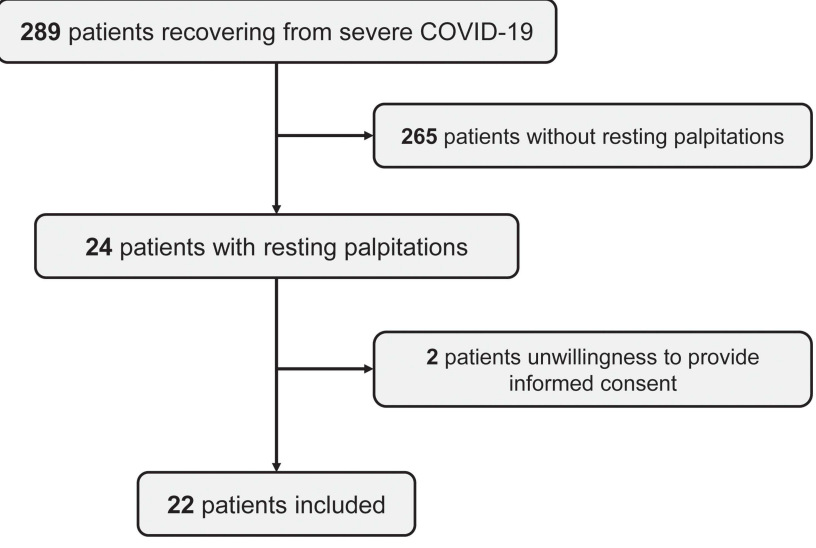

Figure I Flowchart of participant selection. COVID-19=coronavirus disease 2019.
2 patients who refused to provide informed consent, 22 patients were included in this prospective cohort study.

The details of all the characteristics are presented in Table 1. In total, the median age was 52 years (IQR, 44-62 years), 12/22 (54.5\%) patients were female, and the median length of hospital stay was 24 days (IQR, 19-28 days). Half of these patients had chronic diseases, including 8/22 (36.3\%) with hypertension, $3 / 22(13.6 \%)$ with diabetes, $3 / 22$ (13.6\%) with chronic renal disease, $2 / 22(9.1 \%)$ with coronary heart disease, $1 / 22(4.5 \%)$ with chronic heart failure, $1 / 22(4.5 \%)$ with chronic obstructive pulmonary disease, and $1 / 22(4.5 \%)$ with cancer. Many patients presented with organ function

Table I Clinical Characteristics

\begin{tabular}{|c|c|}
\hline Characteristics & All Patients $(n=22)$ \\
\hline Age (years), median (IQR) & $52(44-62)$ \\
\hline Female, n (\%) & $12(54.5)$ \\
\hline Hospital stay (d), median (IQR) & $24(19-28)$ \\
\hline $\begin{array}{l}\text { Chronic medical illness, n (\%) } \\
\text { Hypertension } \\
\text { Diabetes } \\
\text { Chronic renal disease } \\
\text { Coronary heart disease } \\
\text { Chronic heart failure } \\
\text { Chronic obstructive pulmonary disease } \\
\text { Cancer }\end{array}$ & $\begin{array}{l}\text { II }(50) \\
8(36.3) \\
3(13.6) \\
3(13.6) \\
2(9.1) \\
\text { I }(4.5) \\
\text { I }(4.5) \\
\text { I }(4.5)\end{array}$ \\
\hline $\begin{array}{l}\text { Complications, } \mathbf{n}(\%) \\
\text { Acute respiratory distress syndrome } \\
\text { Acute myocardial injury } \\
\text { Acute liver injury } \\
\text { Acute kidney injury } \\
\text { Coagulopathy }\end{array}$ & $\begin{array}{l}12(54.5) \\
4(18.2) \\
4(18.2) \\
3(13.6) \\
2(9.1) \\
2(9.1)\end{array}$ \\
\hline $\begin{array}{l}\text { Treatment, } \mathbf{n}(\%) \\
\text { Antiviral treatment } \\
\text { Antibiotic treatment } \\
\text { Glucocorticoids } \\
\text { Immunoglobulin therapy } \\
\text { Noninvasive ventilation* } \\
\text { Continuous renal replacement therapy }\end{array}$ & $\begin{array}{l}19(86.4) \\
17(77.3) \\
15(68.2) \\
15(68.2) \\
4(18.2) \\
1(4.5)\end{array}$ \\
\hline $\begin{array}{l}\text { Palpitation and HADS scores } \\
\text { Palpitations score, median (IQR) } \\
\text { HADS-A score, median (IQR) } \\
\text { HADS-D score, median (IQR) } \\
\text { Anxiety (HADS-A } \geq 8), \text { n (\%) } \\
\text { Depression (HADS-D } \geq 8), \text { n (\%) }\end{array}$ & $\begin{array}{c}3(2-4) \\
9.5(6.75-14) \\
8(5-10.25) \\
16(68.2) \\
13(59.1)\end{array}$ \\
\hline
\end{tabular}

Notes: *There were no patients on invasive ventilation or extracorporeal membrane oxygenation.

Abbreviations: HADS, the Hospital Anxiety and Depression Scale; HADS-A, HADS anxiety; HADS-D, HADS depression; IQR, interquartile range. 


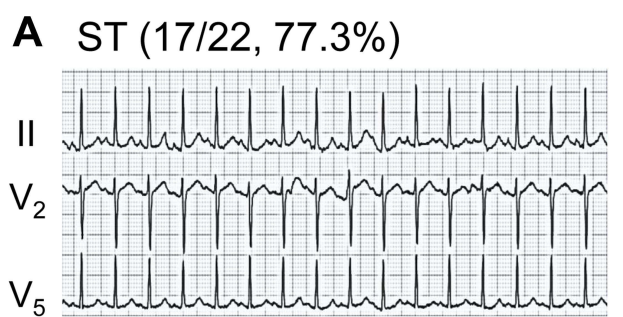

C $\operatorname{AT}(1 / 22,4.5 \%)$
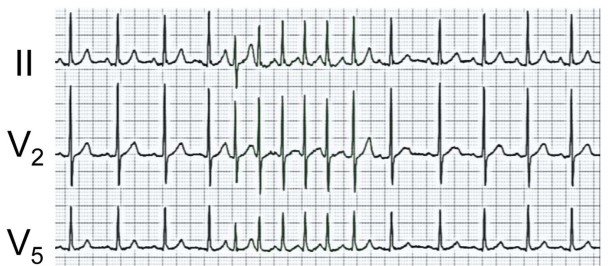

B $\operatorname{APC}(2 / 22,9.1 \%)$

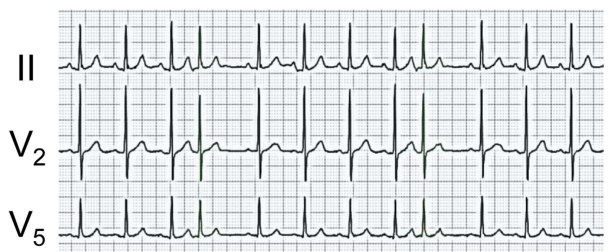

D $\operatorname{VPC}(1 / 22,4.5 \%)$

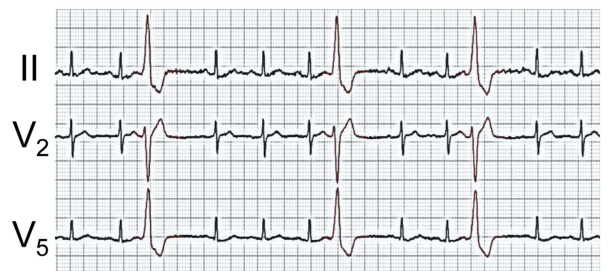

Figure 2 Representative ECG examples of cardiac arrhythmias, including sinus tachycardia (ST) (A), atrial premature contraction (APC) (B), atrial tachycardia (AT) (C), and ventricular premature contraction (VPC) (D).

damage during hospitalization, including 4/22 (18.2\%) with acute respiratory distress syndrome, 4/22 (18.2\%) with acute myocardial injury, 3/22 (13.6\%) with acute liver injury, 2/22 (9.1\%) with acute renal injury, and 2/22 (9.1\%) with coagulopathy. For treatment, 19/22 (86.4\%), 17/22 (77.3\%), 15/22 $(68.2 \%)$ and 15/22 (68.2\%) patients received antiviral drugs, antibiotics, glucocorticoids, and immunoglobulin, respectively. 4/22 (18.2\%) patients used noninvasive mechanical ventilation, and $1 / 22(4.5 \%)$ patient received continuous renal replacement therapy. No patients underwent invasive mechanical ventilation or extracorporeal membrane oxygenation.

\section{Analysis of 2-Hour Holter Monitoring}

Two-hour Holter monitoring showed that 18/22 (81.8\%) patients had cardiac arrhythmias. Figure $2 \mathrm{~A}-\mathrm{D}$ shows representative ECG examples of cardiac arrhythmias. The most common arrhythmia was sinus tachycardia, which occurred in 17/22 (77.3\%) patients. The median maximum heart rate during monitoring was 108.5 bpm (IQR, 101$114 \mathrm{bpm})$. However, patients with sinus tachycardia showed a similar palpitation score as those without sinus tachycardia (median [IQR], 3 [2.5-4] vs 2 [1.5-3.5], $P=0.23$ ) (Figure $3 \mathrm{~A}$ ). In addition, there was no significant relationship between maximum heart rate and palpitation score among the 22 patients $(r=0.323 ; 95 \%$ CI, $-0.11-$ $0.66 ; P=0.14$ ) (Figure 3B). Other arrhythmias included atrial premature contraction in $2 / 22(9.1 \%)$ patients, atrial tachycardia in $1 / 22(4.5 \%)$ patient, and ventricular premature contraction in $1 / 22(4.5 \%)$ patient. No bradyarrhythmias were detected.
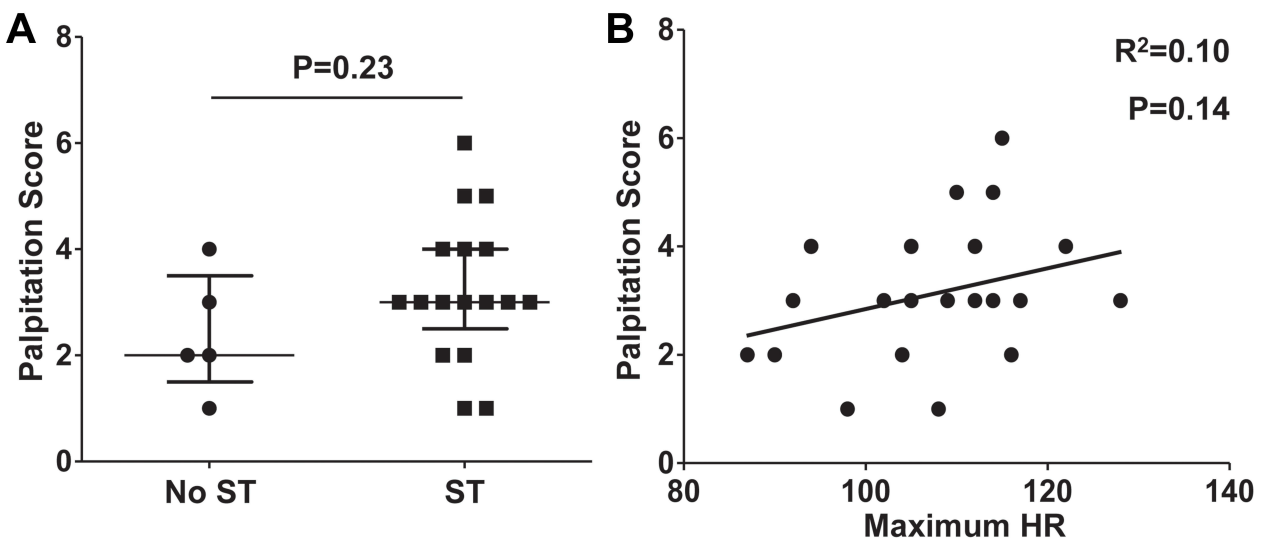

Figure 3 Frequency of palpitation episodes in patients with vs without sinus tachycardia (ST) (A) and correlation analysis of maximum heart rate and frequency of palpitation episodes (B). 

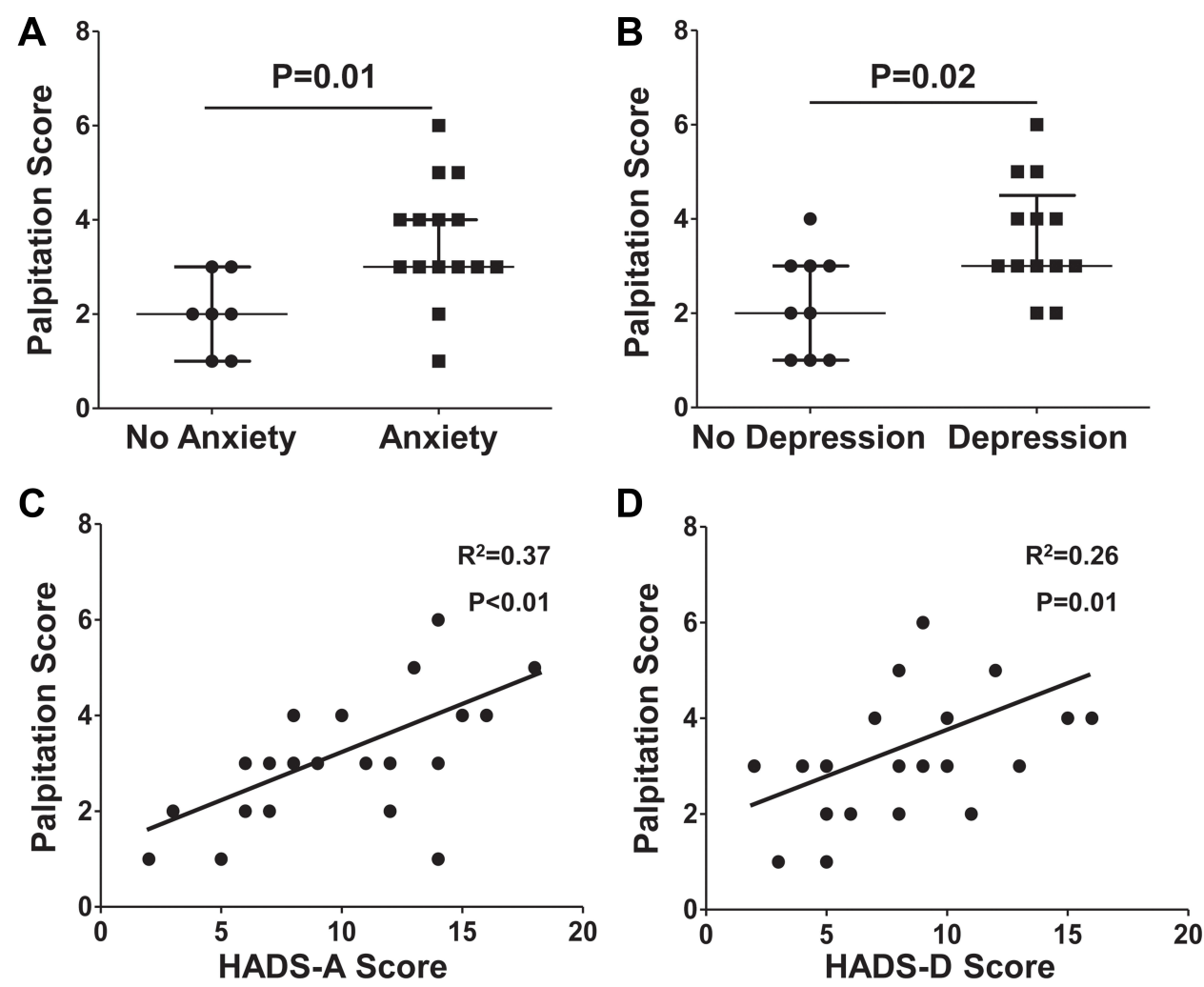

Figure 4 Frequency of palpitation episodes in patients with vs without anxiety (A) or depression (B), and correlation analysis of anxiety (C) or depression (D) levels and frequency of palpitation episodes.

\section{Palpitation Score, HADS Score, and the Correlations Between Them}

The medians of the participants' palpitation score, HADS-A score, and HADS-D score were 3 (IQR, 2-4), 9.5 (IQR, 6.7514), and 8 (IQR, 5-10.25), respectively. Among the 22 patients, $15(68.2 \%)$ had anxiety symptoms (HADS-A $\geq 8$ ), and $13(59.1 \%)$ had depression symptoms (HADS-D $\geq 8$ ) (Table 1). Patients with anxiety symptoms (HADS-A $\geq 8$ ) were associated with higher palpitation scores than patients without anxiety (median [IQR], 3 [3-4] vs 2 [1-3], $P=0.01$ ) (Figure 4A). Similarly, the palpitation score was also significantly higher in patients with depression (HADS-D $\geq 8$ ) than in those without depression (median [IQR], 3 [3-4.5] vs 2 [1-3], $P=0.02$ ) (Figure 4B). In addition, both the HADS-A score $(r=0.609 ; 95 \% \mathrm{CI}, 0.25-0.82 ; P<0.01)$ and HADS-D score $(r=0.516 ; 95 \%$ CI, $0.12-0.77 ; P=0.01)$ were positively related to the palpitation score (Figure $4 \mathrm{C}$ and $\mathrm{D}$ ).

\section{Discussion}

To our knowledge, this is the first study to investigate the correlation between psychological distress and resting palpitations in patients who recovered from severe COVID-19. The major findings of the present study are as follows: (1) symptoms of resting palpitations are not uncommon (8.3\%) among patients who recovered from severe COVID-19; (2) sinus tachycardia is the most common (77.3\%) arrhythmia in recovered patients with resting palpitations; and (3) anxiety (68.2\%) and depression (59.1\%) are also prevalent among these patients, and strong correlations were observed between the psychological burden and the frequency of palpitation episodes.

Infection with SARS-CoV-2 may lead to prolonged and persistent symptoms. Several studies have reported that palpitation is one of the common symptoms in survivors of COVID-19 after discharge. ${ }^{15,16}$ And the prevalence of palpitation symptoms are markedly higher in severe patients than non-severe patients. ${ }^{16}$ In the present study, we found that $24(8.3 \%)$ of the 289 recovered patients had resting palpitation symptoms, consistent with previous studies. It should be noted that all the included patients in the present study denied having previous similar episodes of palpitations. Palpitation symptoms occurred during hospitalization and remained after recovery from severe COVID-19.

Palpitation is a common symptom and often poses clinical challenges due to its wide differential diagnosis. ${ }^{17}$ Cardiac 
arrhythmias and psychological distress are the most common causes in patients presenting with palpitations. ${ }^{7}$ The results of two-hour resting ECG monitoring in this study showed that the large majority of the enrolled patients (81.8\%) had cardiac arrhythmias. Among the arrhythmias, the most common was sinus tachycardia (77.3\%). However, patients with sinus tachycardia had a similar frequency of palpitation episodes compared to those without sinus tachycardia, suggesting that there might be other factors, in addition to sinus tachycardia, affecting palpitation episodes. It has been found in some cases that thyroid dysfunction occurs after the resolution of COVID-19 and contributes palpitation symptoms. ${ }^{18}$ However, none of the recovered patients in this study had any active diseases that might cause palpitation symptoms, such as thyroid dysfunction, haematological disorders, or severe heart failure (left ventricular ejection fraction $<30 \%$ ).

As described above, in addition to arrhythmias, psychological distress is another common cause of palpitations. The prevalence of anxiety and depression was detected by using the HADS-A and HADS-D, respectively, in this study. The results showed that $68.2 \%$ of patients showed anxiety symptoms (HADS-A $\geq 8$ ), while $59.1 \%$ showed depression symptoms (HADS-D $\geq 8$ ). Given the relatively high mortality rate associated with this novel disease, it was not surprising that substantial panic and stress were caused in the early stages of the COVID-19 outbreak. Several studies in China have investigated mental problems in not only COVID-19 patients ${ }^{19,20}$ but also COVID-19-negative patients ${ }^{21}$ and health care workers. ${ }^{22}$ These studies also reported that people in Wuhan or Hubei Province were prone to higher levels of psychological distress than people in other regions of China. ${ }^{21}$ Female sex, disease duration, levels of inflammatory markers and self-perceived illness severity were proposed to be factors that could predict the severity of patients' mental symptoms. ${ }^{20}$ In the present cohort study, all patients recovered from severe COVID-19 in Wuhan, China, with a high incidence of various complications (54.5\%) and very long hospital stays (median [IQR] 24 days [19-28 days]), which might lead to both physical deconditioning and psychological distress.

Psychological distress, such as anxiety and depressionrelated symptomatology, was frequent among patients with complaints of palpitations. Alijaniha et $\mathrm{al}^{8}$ showed that $85.4 \%$ of patients with palpitations had psychological symptoms, and this frequency was twice that of the healthy group (43.6\%). Another study investigating the aetiologies of palpitations suggested that up to one-third of the patients presenting with palpitations were finally identified to have palpitations caused by psychological symptoms. ${ }^{23}$ On the other hand, symptoms of resting palpitations were also one of the most common somatic symptoms associated with anxiety and depression. ${ }^{24} \mathrm{~A}$ recent study by Yuan et $\mathrm{al}^{25}$ found that somatic symptoms were related to the levels of anxiety during the early stages of the COVID-19 outbreak. Consistent with previous studies, we found that patients with anxiety/depression had a higher frequency of palpitation episodes than those without anxiety/depression. Moreover, a close positive correlation between the levels of anxiety/depression and the frequency of palpitation episodes was observed in the present study. These findings suggest that anxiety and depression might be responsible, at least in part, for resting palpitation symptoms in recovered COVID-19 patients. Importantly, a recent retrospective study from the Mayo Clinic showed that anxiety and depression are the most common comorbid conditions in patients with inappropriate sinus tachycardia, ${ }^{26}$ proposing a possible link between psychological distress and sinus tachycardia.

\section{Limitations}

Our study has several limitations. First, accumulating evidence has shown that a minority of COVID-19 patients have cardiac complications, including acute myocardial injury, acute myocardial infarction, and myocarditis, ${ }^{27,28}$ which might result in cardiac structural and electrical changes, causing arrhythmia and palpitation symptoms. However, these effects were not investigated in this study, as only 4 of the enrolled patients had a mild elevation of high-sensitivity troponin I. Second, pulmonary function was not evaluated in the present study since this examination was not available to COVID-19 patients in our hospital, even to those who had just recovered. Indeed, impaired pulmonary function has been reported in recovered COVID-19 and severe acute respiratory syndrome (SARS) patients and affects patients' exercise capacity. ${ }^{29,30}$ However, none of the enrolled participants complained of any shortness of breath or dyspnoea when they were at rest. Therefore, we speculated that the effects of impaired pulmonary function on resting palpitation symptoms were negligible. Third, since only a small number of patients received psychological guidance due to the relatively limited number of trained psychiatrists, the therapeutic effects of psychological counselling on palpitation symptoms were not evaluated in the present study. Finally, the sample size of the study was too small. Studies with larger populations and in multiple centres are warranted.

\section{Conclusions}

Symptoms of resting palpitations, manifested mainly by sinus tachycardia, are not uncommon in patients who 
recovered from severe COVID-19. Anxiety and depression may contribute, at least in part, to resting palpitation symptoms. Since an accurate aetiology leads to an effective treatment, it is highly recommended that psychiatric assessments be performed in recovered COVID-19 patients with resting palpitations after a negative evaluation by a cardiologist. In addition, previous studies in recovered SARS patients have shown that palpitation symptoms may last for a long time after discharge. ${ }^{28}$ Recent studies also show that some COVID-19 patients still suffer from palpitation symptoms even 3 months after discharge. ${ }^{3}$ Considering the causal relationship between psychological distress and palpitation symptoms, future studies are warranted to evaluate whether psychological guidance could shorten the course of palpitation symptoms in recovered COVID-19 patients.

\section{Compliance with Ethical Guidelines}

The study protocol was approved by the ethics committees of Renmin Hospital of Wuhan University and Wuhan Hankou Hospital. All procedures performed in studies involving human participants were in accordance with the ethical standards of the institutional and/or national research committee and with the 1975 Helsinki Declaration and its later amendments or comparable ethical standards. Informed consent was obtained from all individual participants included in the study.

\section{Author Contributions}

All authors contributed to data analysis, drafting or revising the article, gave final approval of the version to be published, agreed to the submitted journal, and agree to be accountable for all aspects of the work.

\section{Funding}

This work was supported by grants from the Foundation of Xinjiang Science and Technology Support Project (2020E0274), and from the Nature Science Foundation of Hubei Province (2021CFB440).

\section{Disclosure}

The authors have no conflicts of interest to declare.

\section{References}

1. Wang $\mathrm{X}, \mathrm{Xu} \mathrm{H}$, Jiang $\mathrm{H}$, et al. Clinical features and outcomes of discharged coronavirus disease 2019 patients: a prospective cohort study. QJM. 2020;113(9):657-665. doi:10.1093/qjmed/hcaa178
2. Shi S, Qin M, Cai Y, et al. Characteristics and clinical significance of myocardial injury in patients with severe coronavirus disease 2019. Eur Heart J. 2020;41(22):2070-2079. doi:10.1093/eurheartj/ehaa408

3. Liang L, Yang B, Jiang N, et al. Three-month follow-up study of survivors of Coronavirus disease 2019 after discharge. J Korean Med Sci. 2020;35(47):e418.

4. Puyod JV, Charoensukmongkol P. Effects of workplace rumors and organizational formalization during the COVID-19 pandemic: a case study of universities in the Philippines. Corp Commun. 2021;26 (4):793-812. doi:10.1108/CCIJ-09-2020-0127

5. Vindegaard N, Benros ME. COVID-19 pandemic and mental health consequences: systematic review of the current evidence. Brain Behav Immun. 2020;89:531-542. doi:10.1016/j.bbi.2020.05.048

6. Weerahandi H, Hochman KA, Simon E, et al. Post-discharge health status and symptoms in patients with severe COVID-19. medRxiv. 2020;2020:20172742. doi:10.1101/2020.08.11.20172742

7. Gale CP, Camm A. Assessment of palpitations. BMJ. 2016;352: h5649. doi:10.1136/bmj.h5649

8. Alijaniha F, Noorbala A, Afsharypuor S, et al. Relationship between palpitation and mental health. Iran Red Crescent Med J. 2016;18(3): e22615. doi:10.5812/ircmj.22615

9. Ehlers A, Mayou RA, Sprigings DC, Birkhead J. Psychological and perceptual factors associated with arrhythmias and benign palpitations. Psychosom Med. 2000;62(5):693-702. doi:10.1097/ 00006842-200009000-00014

10. National Health Commission of People's Republic of China. Chinese clinical guidelines for COVID-19 diagnosis and treatment (fifth edition). In Chinese; ; 2020. Available from: http://www.nhc.gov.cn/yzygj/s7653p/ $202002 / 3$ b09b894ac9b4204a79db5b8912d4440/files/ 7260301a393845fc87fcf6dd52965ecb.pdf. Accessed February 04, 2020.

11. Ranieri VM, Rubenfeld GD, Thompson BT, et al.; ARDS Definition Task Force. Acute respiratory distress syndrome: the Berlin definition. JAMA. 2012;307(23):2526-2533. doi:10.1001/jama.2012.5669

12. Shang Y, Liu T, Wei Y, et al. Scoring systems for predicting mortality for severe patients with COVID-19. EClinicalMedicine. 2020;24:100426. doi:10.1016/j.eclinm.2020.100426

13. Fliser D, Laville M, Covic A, et al.; Ad-hoc working group of ERBP. A European Renal Best Practice (ERBP) position statement on the Kidney Disease Improving Global Outcomes (KDIGO) clinical practice guidelines on acute kidney injury: part 1: definitions, conservative management and contrast-induced nephropathy. Nephrol Dial Transplant. 2012;27(12):4263-4272. doi:10.1093/ndt/gfs375

14. Zigmond AS, Snaith RP. The hospital anxiety and depression scale. Acta Psychiatr Scand. 1983;67(6):361-370. doi:10.1111/j.16000447.1983.tb09716.x

15. Huang Y, Pinto MD, Borelli JL, et al. COVID symptoms, symptom Clusters, and predictors for becoming a long-hauler: looking for clarity in the Haze of the pandemic. medRxiv. 2021;2021. doi:10.1101/2021.03.03.21252086.

16. Qin W, Chen S, Zhang Y, et al. Diffusion capacity abnormalities for carbon monoxide in patients with COVID-19 at 3-month follow-up. Eur Respir J. 2021;58(1):2003677. doi:10.1183/13993003.03677-2020

17. Thavendiranathan P, Bagai A, Khoo C, Dorian P, Choudhry NK. Does this patient with palpitations have a cardiac arrhythmia? JAMA. 2009;302(19):2135-2143. doi:10.1001/jama.2009.1673

18. Brancatella A, Ricci D, Cappellani D, et al. Is subacute thyroiditis an underestimated manifestation of SARS-CoV-2 infection? Insights from a case series. J Clin Endocrinol Metab. 2020;105(10): dgaa537. doi:10.1210/clinem/dgaa537

19. Bo HX, Li W, Yang Y, et al. Posttraumatic stress symptoms and attitude toward crisis mental health services among clinically stable patients with COVID-19 in China. Psychol Med. 2020;27:1-2. doi:10.1017/S0033291720000999

20. Hu Y, Chen Y, Zheng Y, et al. Factors related to mental health of inpatients with COVID-19 in Wuhan, China. Brain Behav Immun. 2020;89:587-593. doi:10.1016/j.bbi.2020.07.016 
21. Qiu J, Shen B, Zhao M, Wang Z, Xie B, Xu Y. A nationwide survey of psychological distress among Chinese people in the COVID-19 epidemic: implications and policy recommendations. Gen Psychiatr. 2020;33(2):e100213. doi:10.1136/gpsych-2020-100213

22. Lai J, Ma S, Wang Y, et al. Factors associated with mental health outcomes among health care workers exposed to Coronavirus disease 2019. JAMA netw open. 2020;3(3):e203976. doi:10.1001/jamanetworkopen.2020.3976

23. Weber BE, Kapoor WN. Evaluation and outcomes of patients with palpitations. Am J Med. 1996;100(2):138-148. doi:10.1016/s00029343(97)89451-x

24. Haug TT, Mykletun A, Dahl AA. The association between anxiety, depression, and somatic symptoms in a large population: the HUNT-II study. Psychosom Med. 2004;66(6):845-851. doi:10.1097/ 01.psy.0000145823.85658.0c

25. Yuan S, Liao Z, Huang H, et al. Comparison of the indicators of psychological stress in the population of Hubei Province and Non-Endemic Provinces in China during two weeks during the Coronavirus disease 2019 (COVID-19) outbreak in February 2020. Med Sci Monit. 2020;26:e923767. doi:10.12659/MSM.923767
26. Shabtaie SA, Witt CM, Asirvatham S. Natural history and clinical outcomes of inappropriate sinus tachycardia. $J$ Cardiovasc Electrophysiol. 2020;31(1):137-143. doi:10.1111/jce.14288

27. Stefanini GG, Montorfano M, Trabattoni D, et al. ST-elevation myocardial infarction in patients with COVID-19: clinical and angiographic outcomes. Circulation. 2020;141(25):2113-2116. doi:10.1161/CIRCULATIONAHA.120.047525

28. Lau ST, Yu WC, Mok NS, Tsui PT, Tong WL, Cheng SW. Tachycardia amongst subjects recovering from severe acute respiratory syndrome (SARS). Int $J$ Cardiol. 2005;100(1):167-169. doi:10.1016/j.ijcard.2004.06.022

29. Chen R, Gao Y, Chen M, et al. Impaired pulmonary function in discharged patients with COVID-19: more work ahead. Eur Respir J. 2020;56(1):2002194. doi:10.1183/13993003.02194-2020

30. Hui DS, Joynt GM, Wong KT, et al. Impact of severe acute respiratory syndrome (SARS) on pulmonary function, functional capacity and quality of life in a cohort of survivors. Thorax. 2005;60 (5):401-409. doi:10.1136/thx.2004.030205

\section{Publish your work in this journal}

The International Journal of General Medicine is an international, peer-reviewed open-access journal that focuses on general and internal medicine, pathogenesis, epidemiology, diagnosis, monitoring and treatment protocols. The journal is characterized by the rapid reporting of reviews, original research and clinical studies across all disease areas. The manuscript management system is completely online and includes a very quick and fair peer-review system, which is all easy to use. Visit http://www.dovepress.com/ testimonials.php to read real quotes from published authors. 